\title{
Carbapenem-resistant Enterobacteriaceae
}

\section{Fatma ESER ${ }^{1}$}

${ }^{1}$ Department of Infectious Disease and Clinical Microbiology, Diskapi Yildirim Beyazit Training and Research Hospital, Ankara, Turkey.

\section{ABSTRACT}

Carbapenem-resistant Enterobacteriaceae (CRE) is a bacterial family difficult to treat with high resistance rates to broad-spectrum antibiotics. Patients following up in long-term-care facilities are the most affected by CRE infections and colonization. Carbapenem resistance rates are increasing despite all precautions, and the infections caused by CRE continues with high mortality. CRE surveillance is recommended by the Centers for Disease Control and Prevention (CDC) related to characteristics of health care facilities, point prevalence, or active surveillance. Mechanical ventilation, urinary or intravenous catheters, and long-term antibiotic use are the main risk factors for CRE infections. Treatment options are limited with older agents and mainly depend on aminoglycosides, polymyxins, and fosfomycin. Moreover, combination therapies and high-dose regimens are the other salvage strategies. This review study examined the epidemiology, precaution strategies, and treatment for CRE infections.

Key words: Carbapenem-resistant, Enterobacteriaceae, nosocomial infection

\section{INTRODUCTION}

Members of Enterobacteriaceae family; particularly Klebsiella pneumoniae, cause serious and life-threatening infections that have a significant mortality rate. Moreover, the treatment options are becoming limited with increasing resistance. The health care-related infections due to carbapenem-resistant Enterobacteriaceae (CRE) have been reported increasingly from all over the world in recent years (1-3). The CRE infections were first reported in the 1990s, but have reached the levels that can cause problems in clinical practice (4). Recent studies showed that exposure to carbapenem-resistant (CR) K. penumoniae is independently related to in-hospital mortality, and mortality rates are significantly higher in patients who develop CRE infections $(1,2,5,6)$.

\section{Carbapenem-resistant Enterobacteriaceae}

Previously resistance of members of Enterobacteriaceae family to carbapenem was defined as being insensitive to doripenem, meropenem, or imipenem, and also being resistant to all third-generation cephalosporins. However, in November 2015, ertapenem resistance was added to the list, and being resistant to all cephalosporins was removed from the definition (7). The CRE control guide of the Centers for Disease Control and Prevention (CDC) defined the CRE resistance as being resistant to any carbapenem antibiotic (i.e., minimum inhibitory concentrations of $\geq 4 \mu \mathrm{g} / \mathrm{mL}$ for doripenem, meropenem, or imipenem OR $\geq 2 \mu \mathrm{g} / \mathrm{ml}$ for ertapenem) or documentation of carbapenemase production. Further, the bacteria that are intrinsically resistant to imipenem (i.e., Morganella morganii, Proteus spp., Providencia spp.) should be resistant to non-imipenem carbapenems, and one of the polymerase chain reaction, modified Hodge test, Carba NP, or metallo- $\beta$-lactamase tests should be used to determine carbapenemase production (7). The breakpoints for carbapenem resistance according to the European Committee on Antimicrobial Susceptibility Testing and the Clinical \& Laboratory Standards Institute (CLSI) are presented in Table $1(8,9)$.

\section{Carbapenemases}

Currently the most important mechanism for the development of carbapenem resistance is the production of the enzymes called "carbapenemases" by the microorganisms, which hydrolyze carbapenems. Carbapenemases hydrolyze not only carbapenems but also the broad-spectrum antibiotics oxyimino-cephalosporins and cephamycins, and they are 


\begin{tabular}{|c|c|c|c|c|c|c|}
\hline \multirow[t]{3}{*}{ Carbapenem } & \multicolumn{3}{|c|}{ Disk diffusion breakpoints (mm) } & \multicolumn{3}{|c|}{ MIC breakpoints (mg/L) } \\
\hline & \multicolumn{6}{|c|}{ CLSI M100-S22 (9) } \\
\hline & $\mathrm{S}$ & 1 & $\mathrm{R}$ & $S$ & 1 & $\mathrm{R}$ \\
\hline Imipenem & $\geq 23$ & $20-22$ & $\leq 19$ & $\leq 1$ & 2 & $\geq 4$ \\
\hline Meropenem & $\geq 23$ & $20-22$ & $\leq 19$ & $\leq 1$ & 2 & $\geq 4$ \\
\hline Ertapenem & $\geq 22$ & $19-21$ & $\leq 18$ & $\leq 0.5$ & 1 & $\geq 2$ \\
\hline \multirow[t]{3}{*}{ Doripenem } & $\geq 23$ & $20-22$ & $\leq 19$ & $\leq 1$ & 2 & $\geq$ \\
\hline & \multicolumn{6}{|c|}{ EUCAST (8) } \\
\hline & $\mathrm{S}$ & & $\mathrm{R}$ & S & & $\mathrm{R}$ \\
\hline Imipenem & $\geq 22$ & & $\leq 16$ & $\geq 2$ & & $\leq 2$ \\
\hline Meropenem & $\geq 22$ & & $\leq 16$ & $\geq 2$ & & $\leq 2$ \\
\hline Ertapenem & $\geq 25$ & & $\leq 22$ & $\geq 0.5$ & & $\leq 0.5$ \\
\hline Doripenem & $\geq 24$ & & $\leq 21$ & $\geq 1$ & & $\leq 1$ \\
\hline
\end{tabular}

CLSI M100-S22, Clinical \& Laboratory Standards Institute; EUCAST, European Committee on Antimicrobial Susceptibility Testing.

responsible for the resistance to a broad group of antibiotics (10). Carbapenemases are categorized into molecular classes $A, B$, and D according to the classification of beta-lactamase family (11). The distribution of carbapenemases according to microorganisms in which they were identified is presented in Table 2 (11).

\section{Class A Carbapenemases}

They are the enzymes responsible for the carbapenem resistance in Enterobacteriaceae family, particularly K. penumoniae. KPC enzymes were first extracted from K. pneumoniae isolates in many epidemics in the USA, and then they were reported from other gram-negative microorganism all over the world $(12,13)$.

\section{Class B Metallo-Beta-Lactamases}

These enzymes use zinc cation to hydrolyze $\beta$-lactam ring, they are sensitive to chelators such as EDTA, and they are resistant to clavulanic acid, sulbactam, and tazobactam. They are responsible for the resistance to all beta-lactam antibiotics but not the monobactams (14). They belong to five clinically important families: IMP, VIM, SPM, GIM, and SIM. Typically, they can be transmitted by plasmids to Acinetobacter, primarily Pseudomonas aeruginosa, and other nonfermenting gram-negative and enteric bacteria (15).

\section{Class D OXA Carbapenemases}

OXA carbapenemase production leads to resistance to carbapenems and penicillins, but not to cephalosporins. Four families of OXAtype beta-lactamases (OXA-23, OXA-24, OXA-40, and OXA-58) have been described in Acinetobacter baumanniii isolates (16).

\section{CRE Epidemiology}

The prevalence of $C R$ members of Enterobacteriaceae family has increased in the last decade in many geographical regions of the world, including the USA and Europe. The resistance to one of the three carbapenems (imipenem, meropenem, and doripenem) in Enterobacteriaceae isolates, which was reported to the National Health Security Network (NHSN) in 2011, reached 4.2\%. This increase in resistance was mostly reported for K. pneumoniae strains (1.6\%-10.4\%) (17). According to the NHSN, the prevalence of 2009-2010 CR Klebsiella strains has reached 12\% in invasive device-related infections (18).

TABLE 2: Distribution of carbapenemases according to microorganisms (11) .

\begin{tabular}{|c|c|c|c|}
\hline Microorganism & $\begin{array}{c}\text { Class A } \\
(\mathrm{KPC}, \mathrm{GES})\end{array}$ & $\begin{array}{c}\text { Class B } \\
\text { (MBL) }\end{array}$ & $\begin{array}{c}\text { Class D } \\
\text { (OXA) }\end{array}$ \\
\hline Pseudomonas aeroginosa & + & + & + \\
\hline Acinetobacter baumannii & & + & + \\
\hline Klebsiella pneumoniae & + & + & + \\
\hline Klebsiella oxytoca & + & + & \\
\hline Esherichia coli & + & + & + \\
\hline Proteus mirabilis & + & + & + \\
\hline Serratia marscescens & + & + & \\
\hline Entrobacter spp. & + & + & \\
\hline Citrobacter freundii & + & + & \\
\hline Morganella morganii & & + & \\
\hline Salmonella enterica & + & & \\
\hline
\end{tabular}




\begin{tabular}{|c|c|c|c|c|}
\hline Çalışma & Microorganism & Year & $\begin{array}{l}\text { Imipenem } \\
(\%)\end{array}$ & $\begin{array}{c}\text { Meropenem } \\
\text { (\%) }\end{array}$ \\
\hline MYSTIC & Gram negatives & 2007 & 2.4 & 0.7 \\
\hline HITIT-2 & K.pneumoniae & 2009 & 3.2 & \\
\hline СOMPACT & Enterobacteriaceae spp. & 2012 & 2.1 & 1.7 \\
\hline Köseoğlu Eser & Enterobacteriaceae spp. & 2014 & 5.7 & 1.9 \\
\hline
\end{tabular}

In Europe, CRE isolates have been identified in France, Finland, Germany, Italy, Greece, Norway, Poland, and Sweden since 2005 (19-25). In Europe, CRE isolates have become important nosocomial pathogens in countries such as Greece, Italy, Israel, and Turkey. Epidemics, particularly related to CR Klebsiella spp., have been reported more frequently worldwide (3, 26-29). According to the 2011 data in Asia-Pacific countries, the mean resistance rate was $9 \%$ in CR Klebsiellaspp. isolates and ranged between $0 \%$ and 25\% (30). The prevalence rates of (R Klebsiella spp. rates in blood circulation were 68\%, 27\%, and 15\% in European countries Greece, Italy, and Cyprus, respectively, and many epidemics throughout the countries were reported (31). According to the regional resistance surveillance program data, which evaluates the resistance of microorganisms in 12 Asia-Pacific countries, carbapenem resistance was 0\%-25\% (mean 9\%) for $K$. pneumoniae isolates, and 0\%-50\% (mean 26\%) for P. aeuroginosa isolates (30). CR K. pneumoniae was first reported in 2001 in Turkey with an isolated strain, and since then, hospital epidemics due to CR K. pneumoniae were reported $(32,33)$. Carbapenem resistance rates reported in different studies conducted in Turkey are presented in Table 3.

K. pneumoniae carbapenemase-type carbapenemase is endemic in countries close to Turkey, but the mechanism of primary carbapenem resistance in Turkey was found to be related to OXA-type enzymes (particularly OXA-48) (38). OXA-48-producing strains have been reported from the Middle East, India, and Europe $(29,39,33)$. KPCproducing members of Enterobacteriaceae family is considered to be endemic in the USA, Israel, and Greece (12, 26, 40-42).

\section{CRE Surveillance}

Suggestions regarding CRE screening are categorized under two headings as point prevalence and screening of patients with high infection risk. If t no case has been identified in a unit previously, a point prevalence study can determine the CRE frequency in that unit rapidly. High-risk infections, all patients who stayed in the same room, or patients who took service and care from the same health care personnel should be screened. Specimens are generally taken from stool, rectum, perirectal area, and, in specific occasions, wound and urine. Active surveillance systems and chlorhexidine bath are recommended for preventing against CRE infections. Active surveillance defines screening of patients at admission and periodically after then (7).

If a new case is identified at a center where CRE is rarely or never isolated, precautions for contact isolation should be taken, units should be warned about hand hygiene and contact precautions, and educational support should be provided to the units. CDC recommends screening of patients who are epidemiologically relevant for CRE, performing a point prevalence study in the unit in case of identification of CRE, and establishing a cohort from the patients or health personnel. Basic precautions and supportive interventions should be implemented in all emergency and longterm treatments and nursing care applications in hospitals or units that have frequent CRES. CDC has developed a terminology to identify the CRE surveillance and also algorithms for surveillance and precautions. CRE identification is recommended primarily for Klebsiella species and Escherichia coli, and screening for other agents is recommended according to the local data (7).

\section{Precaution strategies}

Precautions for all centers that deliver emergency and long-term health care can be collected under eight headings.

1. Hand hygiene: Hand hygiene should be promoted, adherence to hand hygiene and feedbacks should be monitored, and access points to hand hygiene should be provided and enhanced.

2. Contact precautions: Patients with CRE colonization or infection should be placed in accordance with contact precautions, and strict contact precautions should be implemented. Preventive measures should be taken during transfers to high-risk units; health care personnel should be 
educated; adherence and feedback should be monitored, and follow-up should be continued; and clinicians and infection control committees should be informed.

3. Health care personnel should be educated.

4. The use of invasive equipment should be decreased.

5. Establishing patient and worker cohorts: Health care personnel should be separated even if the patients stay in the same room. If single-occupancy rooms are limited, these patients should be separated as the highest-infection-risk group (e.g., patients with incontinence).

6. The information should be given to or taken from the laboratory.

7. Antibiotic use should be appropriately managed.

8. CRE screening: It is performed for identifying colonized patients in contact with CRE-carrying patients; cultures can be taken from stool, rectum, and perirectal area.

Supportive interventions with basic precautions include active surveillance and chlorhexidine bath application (44).

\section{Risk factors for CRE}

Many studies that evaluated risk factors in patients with CRE colonization and infection revealed that health care and antibiotic use were the most important risk factors. Debilitated patients, hospitalization at an intensive care unit, and using carbapenem, cephalosporin, fluoroquinolone, and vancomycin are among the other risk factors related to $C R K$. pneumoniae colonization and infection $(1,5,27,44)$. A limited number of studies found that endoscopic procedures were also potential risk factors for CRE infections (45-47). The CRE isolation rate from clinical specimens in patients with CRE colonization ranged between $8.8 \%$ and $47 \%$ (48-52), and these positive cultures generally (86\%) represent a real infection (49). The predictive factors for the development of infection in patients with CRE colonization were found as hospitalization at an intensive care unit, application of central venous catheter, exposure to antibiotics, previous invasive surgery, and presence of diabetes (48, 49). The mortality rates in invasive infections such as bloodstream infections due to CREs can reach up to $40 \%$. When this was compared with the invasive infections due to carbapenem-sensitive members of Enterobacteriaceae family, the mortality rate was found to be significantly higher $(1,2,51)$. Age, mechanical ventilation, malignity, heart disease, and staying at an intensive care unit were found to be associated with increased mortality in CR K. pneumoniae infections $(1,5,6)$. Removal of catheter and elimination of infection foci by debridement or drainage were found to be independently associated with the survival (1). Correa et al. compared patients infected with $C R$ and carbapenem-sensitive $K$. pneumoniae, and reported that the mortality rates were higher in patients with $C R K$. pneumoniae (50\% vs $27.5 \%$ ), but the difference was not statistically significant ( $P=0.085$ ) (52). Jiao et al. evaluated the risk factors and mortality indicators for CR K. pneumoniae infection/colonization in their retrospective study. They reported that glycopeptide use, cefoperazone-sulbactam use, and tracheostomy were the independent risk factors for CR K. pneumoniae infection/colonization compared with carbapenem-sensitive K. pneumoniae infection/ colonization. Also, advanced age was reported as an independent risk factor for mortality in CR K. pneumoniae infection/colonization (53).

\section{Treatment}

Infections due to multiresistant gram-negative bacilli are difficult to treat, and progress with treatment failure and high mortality $(54,55)$. An exact treatment regimen could not be established to date due to the lack of randomized trials and differences in patient groups (56). Generally, combination therapies and antibiotics are recommended to be applied in highest available doses in serious infections. Treatment options include carbapenems, aztreonam, colistin, aminoglycosides, tigecycline, fosfomycin, and, if sensitive, then quinolones and trimethoprim sulfamethoxazole $(54,57)$.

According to the current knowledge about the first-line treatment regimens for infections due to $C R K$. pneumoniae, colistin and high-dose meropenem treatment is recommended for bloodstream and pulmonary infections; colistin, high-dose meropenem, and tigecycline are recommended for gastrointestinal system and gallbladder infections; and fosfomycin and high-dose meropenem are recommended for urinary system infections. Alternative treatment regimens include gentamycin, fosfomycin, rifampicin, and high-dose tigecycline as alternative options (57). A study evaluated the treatment success of carbapenem monotherapy in patients infected with carbapenemase-producing Enterobacteriaceae, and found that the success rates were associated with the minimum inhibitory concentration of carbapenem (54). An analysis by Lee and Burgess evaluated combination therapy and monotherapy, and revealed that 49 patients (47\%) took monotherapy and 56 patients (53\%) took combination therapy. Treatment failure was found to be significantly higher in patients that took monotherapy (49\% vs 25\%; $P=0.01$ ), and no significant difference was found between combination therapies (55). 


\section{CONCLUSIONS}

Infections caused by CRE are currently important health care problems that need strict control measures owing to their association with increased mortality and limited treatment alternatives. Health care facilities have been recommended to be aware of the status of surveillance strategies. Appropriate treatment options need to be developed with continuous research.

\section{REFERENCES}

1. Patel G, Huprikar S, Factor SH, Jenkins SG, Calfee DP. Outcomes of carbapenem-resistant Klebsiella pneumoniae infection and the impact of antimicrobial and adjunctive therapies. Infect Control Hosp Epidemiol 2008;29:1099-106.

2. Hussein $K$, Raz-Pasteur A, Finkelstein $R$, et al. Impact of carbapenem resistance on the outcome of patients' hospitalacquired bacteraemia caused by Klebsiella pneumoniae. J Hosp Infect 2013;83:307-13.

3. Dizbay M, Tunccan OG, Karasahin O, Aktas F. Emergence of carbapenem resistant Klebsiella spp. infections in a Turkish university hospital: epidemiology and risk factors J Infect Dev Ctries 2014;8:044-049.

4. Gaynes RP, Culver DH. Resistance to imipenem among selected gram-negative bacilli in the United States. Infect Control Hosp Epidemiol 1992;13:10-4.

5. Schwaber MJ, Klarfeld-Lidji S, Navon-Venezia S, Schwartz D, Leavitt A, Carmeli Y. Predictors of carbapenem-resistant Klebsiella pneumoniae acquisition among hospitalized adults and effect of acquisition on mortality. Antimicrob Agents Chemother 2008;52:1028-33.

6. Daikos GL, Petrikkos P, Psichogiou $M$, et al. Prospective observational study of the impact of VIM-1 metallo-betalactamase on the outcome of patients with Klebsiella pneumoniae bloodstream infections. Antimicrob Agents Chemother 2009;53:1868-73.

7. Facility Guidance for Control of Carbapenem Resistant Enterobacteriaceae (CRE) November 2015 Update. http:// www.cdc.gov/hai/organisms/cre/cre-toolkit/

8. The European Committee on Antimicrobial Susceptibility Testing. Version 4.0, 2014. http://www.eucast.org EUCAST

9. Clinical and Laboratuary Standarts Institute. 2012. Performance strandarts for antimicrobial suspectibility testing: 22th informational suplement document M100-S22. Clinical and Laboratuary Standarts Institute CLSI, Wayne, PA, 2012.

10. Opal SM, Pop-Vicas A. Chapter 19: Molecular mechanisms of antibiotic resistance in bacteria. Principles and Practice of Infectious Diseases, 7. Edition

11. Dizbay M, Karbapenemazlar. Önemli ve Sorunlu Gram-Negatif Bakteri İnfeksiyonları, ed. S. Ulusoy, H. Leblebıcıoglu, and D. Arman. Ankara: Bilimsel Tıp Yayınevi, p 111-26, 2012.

12. Bratu $S$, Landman $D$, Haag $R$ et al. Rapid spread of carbapenem resistant Klebsiella pneumoniae in New York City: A new treat to our antibiotic armamentarium. Arch Intern Med 2005;165:1430.

13. Smith Moland $E$, Hanson ND, Herrera VL, et al. Plasmidmediated carbapenemhydrolysing beta-lactamase, KPC-2, in Klebsiella pneumoniae isolates. J Antimicrob Chemoter 2003;51:711.
14. Walsh TR, Toleman MA, Poirel L et al. Metallo-beta-lactamases: the quiet before the storm? Clin Microbiol Rev 2005;18:305325.

15. Segreti J, Kapell KS, Trenholme GM. In vitro activity of beta lactam drugs and sulbactam against Chlamydia trochomatis. Diagn Microbiol Infect Dis 1992;15:371-373.

16. Cormican MG, Jones RN. Antimicrobial activity of sefotaxim tested against infrequently isolated pathogenic species (unusual pathogens). Diagn Microbiol Infect Dis 1995;22:43-48.

17. Zilberberg MD, Shorr AF. Secular trends in gram-negative resistance among urinary tract infection hospitalizations in the United States, 2000-2009. Infect Control Hosp Epidemiol 2013;34 (9):940-6.

18. Sievert DM, et al., Antimicrobial-resistant pathogens associated with healthcare-associated infections summary of data reported to the National Healthcare Safety Network at the Centers for Disease Control and Prevention, 2009-2010. Infection Control and Hospital Epidemiology 2013; 34(01): 1-14.

19. Naas T, et al., Plasmid-mediated carbapenem-hydrolyzing $\beta$-lactamase KPC in a Klebsiella pneumoniae isolate from France. Antimicrobial Agents and Chemotherapy 2005;49(10): 4423-4424.

20. Woodford N, et al., Arrival of Klebsiella pneumoniae producing KPC carbapenemase in the United Kingdom. Journal of Antimicrobial Chemotherapy 2008;62(6):1261-1264.

21. Samuelsen $\varnothing$, et al., Emergence of clonally related Klebsiella pneumoniae isolates of sequence type 258 producing plasmidmediated KPC carbapenemase in Norway and Sweden. Journal of Antimicrobial Chemotherapy 2009;63(4):654-658.

22. Baraniak A, et al., Emergence of Klebsiella pneumoniae ST258 with KPC-2 in Poland. Antimicrobial Agents and Chemotherapy, 2009;53(10):4565-4567.

23. Giani T, et al., Emergence in Italy of Klebsiella pneumoniae sequence type 258 producing KPC-3 carbapenemase. Journal of Clinical Microbiology 2009;47(11):3793-3794.

24. Osterblad $\mathrm{M}$, et al., First isolations of KPC-2-carrying ST258 Klebsiella pneumoniae strains in Finland, June and August 2009. Euro surveillance: bulletin Europeen sur les maladies transmissibles $=$ European communicable disease bulletin 2008; 14(40): 331-343.

25. Wendt C, et al., First outbreak of Klebsiella pneumoniae carbapenemase (KPC)-producing K. pneumoniae in Germany. European Journal of Clinical Microbiology and Infectious Diseases 2010; 29: 563-570.

26. Dizbay $\mathrm{M}$, et al., Emergence of carbapenem-resistant Klebsiella spp. infections in a Turkish university hospital: epidemiology and risk factors. The Journal of Infection in Developing Countries 2014;8:044-049.

27. Nordmann P, Cuzon G, Naas T. The real threat of Klebsiella pneumoniae carbapenemase-producing bacteria. The Lancet Infectious Diseases 2009;9:228-236.

28. Hussein K, et al., Carbapenem Resistance Among Klebsiella pneumoniae Isolates Risk Factors, Molecular Characteristics, and Susceptibility Patterns. Infection Control and Hospital Epidemiology 2009;30:666-671.

29. Leavitt A, et al., Emergence of KPC-2 and KPC-3 in carbapenemresistant Klebsiella pneumoniae strains in an Israeli hospital. Antimicrobial Agents and Chemotherapy 2007;51(8):30263029. 
30. Grundmann $\mathrm{H}$, et al., Carbapenem-non-susceptible Enterobacteriaceae in Europe: conclusions from a meeting of national experts. Eurosurveillance, 2010.

31. Mendes RE, et al. Regional resistance surveillance program results for twelve Asia-Pacific nations (2011). Antimicrobial Agents and Chemotherapy 2013; AAC. 01121-13.

32. Glasner C, et al. Carbapenemase-producing Enterobacteriaceae in Europe: a survey among national experts from 39 countries, February 2013. Euro Surveillance 2013; 18(28): 20525.

33. Poirel L, Heritier C, Tolun V, Nordmann P. Emergence of oxacillinase-mediated resistance to imipenem in Klebsiella pneumoniae. Antimicrob Agents Chemother 2004;48 (1):15-22.

34. Korten V, Ulusoy S, Zarakolu P, Mete B. Antimiotic resistance surveillance over a 4-year period (2000-2003) in Turkey: results of the MYSTIC Program. Diagn Microbiol Infect Dis 2007;59 (4):453-7.

35. Gur D, Hascelik G, Aydın N et al. Antimicrobial resistance in Gram negative hospital isolates: results of the Turkish HITIT-2 Surveillance Study of 2007. J Chemother 2009;21(4):383-9. PMid:19622455

36. Leblebicioglu H, Cakir N, Celen M, Kurt H, Baris H, Laeuffer J (2012) Comparative activity of carbapenem testing (the COMPACT study) in Turkey. BMC Infect Dis 12:42.

37. Eser OK, Altun Uludağ $H$, Ergin A, Boral B, Sener B, Hasçelik G. Carbapenem resistance in ESBL positive Enterobacteriaceae isolates causing invasive infections. Mikrobiyol Bul 2014;48 (1):59-69

38. Gülmez D, Woodford N, Palepou MF et al. Carbapenem-resistant Escherichia coli and Klebsiella Pneumoniae isolates from Turkey with OXA-48 like carbapenemases and outher membrane protein loss. Int J Antimicrob Agents 2008;31(6):523-6.

39. Carrër A, et al. Spread of OXA-48-positive carbapenem-resistant Klebsiella pneumoniae isolates in Istanbul, Turkey. Antimicrobial Agents and Chemotherapy 2008;52(8):2950-2954

40. Aktaş $Z$, et al. Carbapenem-hydrolyzing oxacillinase, OXA48, persists in Klebsiella pneumoniae in Istanbul, Turkey. Chemotherapy 2008;54(2):101-106.

41. Souli $M$, et al. An outbreak of infection due to $\beta$-lactamase Klebsiella pneumoniae carbapenemase 2-producing $\mathrm{K}$. pneumoniae in a Greek university hospital: molecular characterization, epidemiology, and outcomes. Clinical Infectious Diseases 2010;50(3):364-373.

42. Marchaim $D$, et al. Outcomes and genetic relatedness of carbapenem-resistant enterobacteriaceae at Detroit medical center. Infection Control and Hospital Epidemiology 2011; 32(09): 861-871.

43. Cuzon G, et al. Worldwide diversity of Klebsiella pneumoniae that produce beta-lactamase blaKPC-2 gene. Emerg Infect Dis 2010;16(9):1349-56.

44. "Management of Multidrug-Resistant Organisms in Healthcare Settings, 2006"(http://www.cdc.gov/hicpac/mdro/mdro_toc. html)

45. Wiener-Well $Y$, Rudensky B, Yinnon AM, et al. Carriage rate of carbapenem resistant Klebsiella pneumoniae in hospitalised patients during a national outbreak. J Hosp Infect 2010; 74:344-9.
46. Orsi GB, García-Ferna'ndez A, Giordano A, et al. Risk factors and clinical significance of ertapenem-resistant Klebsiella pneumoniae in hospitalised patients. J Hosp Infect 2011;78 (1):54-8

47. Orsi GB, Bencardino A, Vena A, et al. Patient risk factors for outer membrane permeability and KPC-producing carbapenemresistant Klebsiella pneumoniae isolation: results of a double case-control study. Infection 2013;41 (1):61-7

48. Orsi GB, Venditti M. Carbapenem-resistant Klebsiella pneumoniae transmission associated to endoscopy. Am J Infect Control 2013;41 (9):849-50

49. Borer A, Saidel-Odes L, Eskira S, et al. Risk factors for developing clinical infection with carbapenem-resistant Klebsiella pneumoniae in hospital patients initially only colonized with carbapenem-resistant K.pneumoniae. Am J Infect Control 2012;40 (5):421-5

50. Schechner $V$, Kotlovsky $T$, Kazma $M$, et al. Asymptomatic rectal carriage of bla(KPC) producing carbapenem-resistant Enterobacteriaceae: who is prone to become clinically infected? Clin Microbiol Infect 2013;19 (5):451-6

51. Debby BD, Ganor O, Yasmin M, et al. Epidemiology of carbapenem resistant Klebsiella pneumoniae colonization in an intensive care unit. Eur J Clin Microbiol Infect Dis 2012;31 (8):1811-17

52. Ben-David D, Kordevani R, Keller N, et al. Outcome of carbapenem resistant Klebsiella pneumoniae bloodstream infections. Clin Microbiol Infect 2012;18 (1):54-60

53. Correa L, Martino MD, Siqueira I et al. A hospital-based matched case-control study to identify clinical outcome and risk factors associated with carbapenemresistant Klebsiella pneumoniae infection. BMC Infect Dis 2013;13:80. doi: 10.1186/1471-233413-80.

54. Jiao Y, Qin Y, Liu J, Li Q et al. Risk factors for carbapenemresistant Klebsiella pneumoniae infection/colonization and predictors of mortality: a retrospective study. Pathog Glob Health 2015;109(2):68-74. doi:10.1179/204777321 5Y.0000000004. Epub 2015 Feb 24.

55. Tzouvelekis LS1, Markogiannakis A, Psichogiou M, Tassios PT, Daikos GL Carbapenemases in Klebsiella pneumoniae and other Enterobacteriaceae: an evolving crisis of global dimensionsClin Microbiol Rev 2012;25(4):682-707. doi: 10.1128/CMR.05035-11.

56. GC Lee, DS Burgess. Treatment of Klebsiella pneumoniae carbapenemase (KPC) infections: a review of published case series and case reports. Ann Clin Microbiol Antimicrob 2012 biomedcentral.com

57. Falagas ME, Lourida P, Poulikakos P, Rafailidis PI, Tansarli GS. Antibiotic treatment of infections due to carbapenem-resistant Enterobacteriaceae: systematic evaluation of the available evidence. Antimicrob Agents Chemother 2014;58(2):654-63. doi: 10.1128/AAC.01222-13. Epub 2013 Sep 30.

58. Zavascki AP, Bulitta JB, Landersdorfer CB Combination therapy for carbapenem-resistant Gram-negative bacteria. Expert Rev Anti Infect Ther 2013;11 (12):1333-53. doi: 10.1586/14787210.2013.845523. Epub 2013 Nov 6. 